UDC 579:252

SHAHMURADOV I.A.

Institute of Molecular Biology and Biotechnologies, ANAS,

Azerbaijan, AZ1073, Baku, Matbuat Ave., 2a, e-mail: ilhambaku@gmil.com, (+994-50)3842496

\title{
NOVEL TOOLS FOR THE PREDICTION OF PROMOTERS IN PLANTS AND BACTERIA
}

The promoter is a chromosome region that determines where the transcription of a particular DNA region is initiated. Promoter recognition is important in defining the transcription units responsible for specific pathways and gene regulation. Initiation of transcription is a dynamic partnership between RNA polymerase (RNAP) and promoter.

In nuclear genomes of eukaryote organisms, transcription process is conducted by multiple types of RNA polymerases. In particular, all protein genes and most noncoding RNA genes, as well as DNA regions of unknown functions are transcribed by RNA polymerase II. $30-50 \%$ of all known promoters contain a TATA-box located from 40 to $18 \mathrm{bp}$ upstream of the TSS. However, promoters of many large groups of genes (e.g. housekeeping genes) lack the TATA-box; the corresponding promoters are referred to as TATAless promoters [1-3].

In contrast to eukaryotes, bacteria have a single form of the RNAP core enzyme [4]. However, this RNAP alone is not able to recognize and bind to promoters to initialize transcription. Different $\sigma$ factors are required that temporarily binds the RNAP core enzyme, determine the RNAPpromoter binding specificity and transcription start site (TSS), depending on nutritional or environmental conditions or developmental stage [5, 6].

Bacterial $\sigma$ factors are classified into two families with distinct structure and function, termed as $\sigma^{70}$ and $\sigma^{54}$ in Escherichia coli. While most bacteria possess multiple members of the $\sigma^{70}$ family, they contain a single representative of the $\sigma^{54}$ family, which is involved in nitrogen metabolism. Cyanobacteria lack any $\sigma^{54}$-like factors $[5,7,8]$.

Due to the development of advanced experimental technologies a great progress was made in analysis of gene regulatory sequences [9-11]. However, a detailed experimental exploration of transcripts is still a quite expensive and difficult procedure. Therefore, in addition to experimental efforts, accurate computational identification of putative promoter regions remains an important task of genomics and post-genomics studies.

Over the last decade various promoter pre- diction programs have been developed. Recent studies indicate that there is often no single TSS, but rather a whole transcription start region (TSR) with multiple TSSs $[12,13]$. However, for genome annotation projects predicting TSRs spanning several hundred (from 250 up to 1000) nucleotides is less useful to identify a gene start point. For such tasks, finding the TSSs seems to be more informative.

To date, various computer programs aimed to predict plant promoters have been developed [1418]. In particular, previously we developed the TSSP-TCM program that showed a quite high accuracy of TSS prediction in the test sequences with experimentally validated TSSs: $87.5 \%$ and $84 \%$ for TATA and TATA-less promoters, respectively.

The first attempt to predict bacterial promoters was by position weight matrices (PWM), which relied on the conservation of the -35 and the -10 elements for $\sigma^{70}$, combined with the distribution of the distance between them $[19,20]$. Later, more accurate bacterial promoter prediction tools have been developed [21-28]. Despite these efforts, all these tools tend to produce many false positives or show poor sensitivity, particularly when they are applied to long sequences or whole genomes. Another restriction of these tools is that they are limited to the prediction of $\sigma^{70}$ promoters in the model organism E. coli, and very rarely can extend to other bacterial species. Therefore, novel, more accurate and efficient tools are required for the computational recognition of different classes of promoters in a broader taxonomical scope.

In this paper, two new computer tools, TSSPlant for prediction of plant promoters for RNA polymerase II, and and bTSSfinder for predicting TSSs in E. coli and three cyanobacterial species are briefly described.

\section{Materials and methods}

12,467 TSSs assigned to the annotated protein coding genes of Arabidopsis thaliana and Ory$z a$ sativa japonica were obtained from the Plant Promoter Database (ppdb), version 2.0 [29, http://ppdb.agr.gifu-u.ac.jp/ppdb/cgi-bin/index.cgi]. Using the genome annotations of Arabidopsis 
(https://www.arabidopsis.org/) and rice (http://rapdb.dna.affrc.go.jp/) genomes, the promoter set of $251 \mathrm{bp}$ sequences (200 bp upstream and $51 \mathrm{bp}$ downstream of a TSS) was created. Besides, 567 plant promoter sequences of 251 bp lengths with experimentally validated TSS, including 50 rice and 106 Arabidopsis promoters, were selected from the current release of PlantProm DB (http://www.softberry.com/plantprom2016/). With merging ppdb and PlantProm DB sets, the final set of 12,948 plant promoters was created. Out of them, 11893 promoters (including 426 sequences from the PlantProm DB) were used as the learning set. For testing, we selected 2 sets: Set 1 of 1000 sequences from ppdb only, and Set 2 of 55 promoters only from PlantProm DB. Length of sequences in the Learning set and Test set 1 was $251 \mathrm{bp}$. Promoter sequences of the Set 2 were extended up to $1101 \mathrm{bp}: 1,000 \mathrm{bp}$ upstream of TSS and $101 \mathrm{bp}$ downstream. As a negative dataset, Arabidopsis and rice genomic sequences composed of CDSs and introns were used.

Data on plant transcription factor binding sites (TFBSs) were obtained from the Regsite DB (www.softberry.com; Plant division) that contained 1,976 TFBSs.

Bacterial TSS/promoter sets were created for E. coli K12 MG1655 (RegulonDB, version v8.0), the non-marine cyanobacterium Nostoc sp. PCC 7120 [30], the freshwater cyanobacterium S. elongatus PCC 6301 [31] and the freshwater cyanobacterium Synechocystis sp. PCC 6803 [32]. The final TSS count was as follows: 1) E. coli: 1,544 for $\sigma^{70}$, 140 for $\sigma^{38}, 237$ for $\sigma^{32}, 135$ for $\sigma^{28}, 412$ for $\sigma^{24} ; 2$ ) Nostoc: 11,386, 3) S. elongatus: 1,471 and 4) Synechocystis: 343.

Data on the bacterial TFBSs were obtained from three sources: 2,953 sites for $E$. coli from RegulonDB, 30 cyanobacterial sites from CollecTF (33), and 63 sites from the literature.

To compute Nucleotide Frequency Matrices (NFM) for the core-promoter elements (TATA-box, -35 box, -10 box, etc.), the Expectation Maximization (EM) algorithm [34] was utilized. It was applied also for the promoter classification.

To distinguish promoter and non-promoter sequences, we explored about 30 different sequence characteristics of positive and negative learning sets. Based on the values of Mahalanobis distances [35] of individual characteristics, we selected up to 21 features for different classes of plant [36] and bacterial [37] promoters.
To get recognition function (classifier) parameters giving the best separation of promoter and non-promoter sequences, separately for two promoter classes, we applied Neural Network (NN) technique $[36,37]$.

\section{Results and discussion}

TSSPlant: the plant promoter prediction tool

The program analyzes each sliding window of $251 \mathrm{bp}$ over the query sequence, one nucleotide at a time, where position 201 is assigned to be classified as TSS or non-TSS. The classification is based on a threshold that was computed during the training. Predictions with the score higher than the threshold are marked as putative TSSs. The program performs additional filtering by discarding all but the highest-scoring TSS in intervals of a userspecified length (default is $300 \mathrm{bp}$ ). TSSPlant is available to download as a standalone program at http://www.cbrc.kaust.edu.sa/download/.

We tested our TSS finder on positive and negative sets of TATA and TATA-less promoter classes. For TATA class, we observed very high prediction accuracy, with sensitivity $\simeq 99 \%$, specificity $\simeq 98 \%$, F1 score $\simeq 99 \%$ and Matthews correlation coefficient (MCC) 0.97. In the case of TATA-less promoters, we also achieved quite a good performance (with sensitivity $\simeq 82 \%$, specificity $\simeq 97 \%$, F1 score $\simeq 89 \%$ and MCC 0.83 ).

We compared our TSSPlant predictor with tools available for on-line execution or downloading and run locally: NNPP [38], Proscan [39], EP3 [40] and TSSP (http://www.softberry.com/ berry.phtml). First, we compared NNPP, Proscan, TSSP and TSSPlant tools on short (251 bp) sequences, randomly chosen from TATA and TATAless test sets. For both TATA and TATA-less cases, the accuracy data clearly indicate that TSSPlant has significantly higher prediction accuracy (results not shown). Another tests were performed on long sequences of $1,100 \mathrm{bp}$ regions of 55 plant protein coding genes with experimentally validated TSS collected in PlantProm DB (Table 1). TSSPlant produced the best accuracy $(\mathrm{Sn} \simeq 72 \%$, $\mathrm{F} 1 \simeq 47 \%)$, followed by TSSP $(\mathrm{Sn}=63.6 \%$, $\mathrm{F} 1 \simeq 41.2 \%), \mathrm{NNPP}(\mathrm{Sn}=51 \%, \mathrm{~F} 1 \simeq 31 \%), \mathrm{EP} 3$ $(\mathrm{Sn} \simeq 20 \%, \mathrm{~F} 1 \simeq 31 \%)$ and Proscan $(\mathrm{Sn} \simeq 9 \%$, $\mathrm{F} 1 \simeq 15 \%$ ).

bTSSfinder: the bacterial promoter prediction tool

The largest collection of experimentally vali- 
dated promoters of $E$. coli in RegulonDB was classified into seven different sigma classes $\left(\sigma^{70}, \sigma^{54}\right.$, $\sigma^{38}, \sigma^{32}, \sigma^{28}, \sigma^{24}$ and $\sigma^{19}$ ). Unfortunately, no such classification exists for cyanobacterial promoters. Our preliminary comparison of E. coli and cyanobacterial promoters indicate that there is a level of conservation, based on which we used E. coli PWMs for the classification of cyanobacterial promoters. Based on the inter-phyla orthology (data not shown), we propose the classification for cyanobacterial promoters into five classes: $\sigma^{\mathrm{A}}$ (analogous to $\sigma^{70}$ ), $\sigma^{\mathrm{C}}$ (analogous to $\sigma^{38}$ ), $\sigma^{\mathrm{F}}$ (analogous to $\left.\sigma^{28}\right), \sigma^{\mathrm{G}}$ (analogous to $\sigma^{24}$ ) and $\sigma^{\mathrm{H}}$ (analogous to $\left.\sigma^{32}\right)$.
Using a combination of features for each promoter class, we built $10 \mathrm{NN}$ classifiers, one for each promoter class in $E$. coli and in cyanobacteria. Then, we implemented these models into the bTSSfinder program. The program slides a window of $251 \mathrm{bp}$ over the query sequence, one nucleotide at a time (analogous to TSSPlant tool). bTSSfinder is available standalone and online at http://www.cbrc.kaust.edu.sa/btssfinder.

We tested bTSSfinder on sets for every promoter class in E. coli and cyanobacteria (Table 2). We observed good performance for all promoter classes in E. coli $(251 \mathrm{bp}$, a single search window size). In the case of cyanobacteria, we observed the highest accuracy in $\sigma^{A}$ promoters ( $F_{1}$-score: 0.94$)$.

Table 1. Comparison of four promoter prediction tools assessed on 1,100 bp region of 55 plant protein coding genes with experimentally validated TSS

\begin{tabular}{|c|c|c|c|c|c|c|c|}
\hline Tool & $\begin{array}{c}\text { Genes with } \\
\geq 1 \mathrm{TSSpr}\end{array}$ & $\begin{array}{c}\text { Total number } \\
\text { of TSSpr }\end{array}$ & $\mathrm{TP}^{1}$ & FP & FN & Sn, \% & F1-score, $\%$ \\
\hline TSSPlant & 54 & 115 & 40 & 75 & 15 & 72.7 & 47.1 \\
\hline TSSP & 45 & 105 & 35 & 80 & 20 & 63.6 & 41.2 \\
\hline NNPP & 47 & 122 & 28 & 97 & 27 & 50.9 & 31.1 \\
\hline EP3 $^{2}$ & 16 & 16 & 11 & 5 & 44 & 20.0 & 31.0 \\
\hline Proscan $^{2}$ & 10 & 10 & 5 & 5 & 50 & 9.1 & 15.4 \\
\hline
\end{tabular}

Notes: ${ }^{1}$ Prediction is considered true, if the distance between annotated TSS and predicted TSS (TSSpr) is $50 \mathrm{bp}$ or less. ${ }^{2} \mathrm{EP} 3$ and Proscan programs perform a search for transcription start region (of $250 \mathrm{nt}$ and $400 \mathrm{nt}$, respectively).

Table 2. Comparison of available promoter prediction programs assessed on the $1,100 \mathrm{bp}$ upstream region of 200 E. coli $\sigma^{70}$ promoters with experimentally validated TSSs

\begin{tabular}{|c|c|c|c|c|c|c|}
\hline 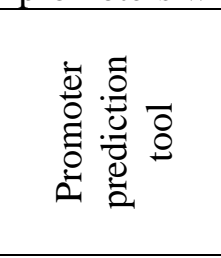 & 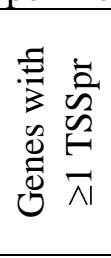 & 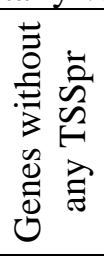 & $\begin{array}{l}0 \\
\Xi \\
D \\
D \\
\vdots \\
0 \\
0 \\
0 \\
0 \\
0 \\
0 \\
0\end{array}$ & 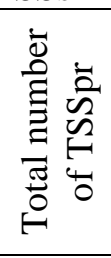 & $\begin{array}{l}\text { do } \\
\text { जी }\end{array}$ & 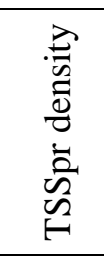 \\
\hline bTTSSfinder & 197 & 3 & 143 & 355 & 71.5 & 620 \\
\hline BPROM & 200 & 0 & 130 & 569 & 65.0 & 386 \\
\hline NNPP2 & 175 & 25 & 109 & 500 & 54.5 & 440 \\
\hline PromPredict & 74 & 126 & 0 & 149 & 0.0 & $1477^{2}$ \\
\hline
\end{tabular}

Notes: TSSan: annotated TSS position 1001), TSSpr: predicted TSS. ${ }^{1}$ Prediction is true, if distance between annotated and predicted TSSs is $50 \mathrm{bp}$ or less. ${ }^{2} \mathrm{But}$, no any true prediction.

We could only evaluate bTSSfinder against previously published promoter prediction tools for $\sigma^{70}$ promoter class in E. coli. For fairness, we assessed all tools on a single testing dataset. The following promoter prediction tools were available for comparison: BPROM (27), NNPP2 (24), and PromPredict (26). All other promoter prediction tools that we checked were no longer available. Results of the comparison for short (251 bp) se- quences clearly indicate that bTSSfinder has significantly higher prediction accuracy (data not shown). However, using short sequences to predict TSSs is not sufficient in evaluating the accuracy and efficiency (especially the real false positive rate) of a prediction tool. It should also be tested on longer sequences. We run the four programs on longer DNA sequences to search for putative $\sigma^{70}$ TSSs in 200 test sequences of $1,101 \mathrm{bp}$ from $E$. coli. As presented in Table 2, bTSSfinder produced the best 
performance $(\mathrm{Sn} \simeq 72 \%, \mathrm{~F} 1 \simeq 52 \%)$, followed by $\mathrm{BPROM}(\mathrm{Sn}=65 \%, \mathrm{~F} 1 \simeq 34 \%)$ and NNPP2 $(\mathrm{Sn}$ $\simeq 54 \%, \mathrm{~F} 1 \simeq 33 \%$ ). Surprisingly, PromPredict failed to produce a single true positive prediction $(\mathrm{Se}=0, \mathrm{~F} 1=0)$.

This publication is based upon work supported by

(1) the King Abdullah University of Science and Tech- nology (KAUST) Office of Sponsored Research (OSR) under Awards No URF/1/1976-02 and FCS/1/2448-01; (2) Grant EIF-2010-1(1)-40/27-3 from the Science Development Foundation under the President of the Republic of Azerbaijan; (3) Science and Technology Center of Ukraine, (STCU) Project \#6154; (4) Azerbaijan National Academy of Sciences.

\section{References}

1. Solovyev V.V., Shahmuradov I.A., Salamov A.A. Identification of promoter regions and regulatory sites. In: Computational Biology of Transcription Factor Binding (Methods in Molecular Biology). Editor: Istvan Ladunga. Springer Science+Business Media, Humana Press, 2010, 674, Chapter 5. doi: 10.1007/978-1-60761-854-6_5.

2. Hernandez-Garcia C.M., Finer J.J. Identification and validation of promoters and cis-acting regulatory elements // Plant Science. - 2014. - V. 217-218. - P. 109-119.

3. Roy A.L., Singer D.S. Core promoters in transcription: old problem, new insights // Trends Biochem Sci. - 2015. - V. 40. P. $165-171$.

4. Schneider G.J., Hasekorn R. RNA polymerase subunit homology among cyanobacteria, other eubacteria and archaebacteria // J Bacteriol. - 1988. - V. 170. - P. 4136-4140.

5. Imamura S., Asayama M. Sigma factors for cyanobacterial transcription // Gene Regul Syst Bio. - 2009. - V. 3. - P. 65-87.

6. Ruff E.F., Record M.T., Jr., Artsimovitch I. Initial events in bacterial transcription initiation // Biomolecules. - 2015. - V. 5. P. 1035-1062.

7. Wosten M.M. Eubacterial sigma-factors // FEMS Microbiol Rev. - 1998. - V. 22. - P. 127-150.

8. Gruber T.M., Gross C.A. Multiple sigma subunits and the partitioning of bacterial transcription space // Annu Rev Microbiol. 2003. - V. 57. - P. 441-466.

9. Mundade R., Ozer H.G., Wei H., Prabhu L., Lu T. Role of ChIP-seq in the discovery of transcription factor binding sites, differential gene regulation mechanism, epigenetic marks and beyond // Cell Cycle. - 2014. - V. 13. - P. 2847-2852.

10. Suryamohan K., Halfon M.S. Identifying transcriptional cis-regulatory modules in animal genomes // Wiley Interdiscip Rev Dev Biol. - 2015. - V. 4. - P. 59-84.

11. Levati E., Sartini S., Ottonello S., Montanini B. Dry and wet approaches for genome-wide functional annotation of conventional and unconventional transcriptional activators // Comput Struct Biotechnol J. - 2016. - V. 14. - P. 262-270.

12. Frith M.C., Valen E., Krogh A., Hayashizaki Y., Carninci P., Sandelin A.A code for transcription initiation in mammalian genomes // Genome Res. - 2008. - V. 18. - P. 1-12.

13. Abeel T., Van de Peer Y., Saeys Y. Toward a gold standard for promoter prediction evaluation // Bioinformatics. - 2009. V. 25. - P. i313-i320.

14. Scherf M., Klingenhoff A., Werner T. Highly specific localization of promoter regions in large genomic sequences by PromoterInspector: a novel context analysis approach // J. Mol. Biol. - 2000. - V. 297. - P. 599-606.

15. Ohler U., Niemann H. Identification and analysis of eukaryotic promoters: recent computational approaches // Trends Genet. 2001. - V. 17. - P. 56-60.

16. Shahmuradov I.A., Solovyev V.V., Gammerman A.J. Plant promoter prediction with confidence estimation // Nucleic Acids Res. - 2005. - V. 33. - P. 1069-1076.

17. Azad A.K.M., Shahid S., Noman N., Lee H. Prediction of plant promoters based on hexamers and random triplet pair analysis // Algorithms for Molecular Biology. - 2011. - V. 6. - P. 19.

18. Zuo Y-C., Li Q-Z. Identification of TATA and TATA-less promoters in plant genomes by integrating diversity measure, GCSkew and DNA geometric flexibility // Genomics. - 2011. - V. 97. - P. 112-120.

19. Hertz G.Z., Stormo G.D. Escherichia coli promoter sequences: analysis and prediction. Methods in enzymology. - 1996. V. 273. - P. 30-42.

20. Huerta A.M., Collado-Vides J. Sigma70 promoters in Escherichia coli: specific transcription in dense regions of overlapping promoter-like signals // J Mol Biol. - 2003. - V. 333. - P. 261-278.

21. Gordon L., Chervonenkis A.Y., Gammerman A.J., Shahmuradov I.A., Solovyev V.V. Sequence alignment kernel for recognition of promoter regions // Bioinformatics. - 2003. - V. 19. - P. 1964-1971.

22. Gordon J.J., Towsey M.W., Hogan J.M., Mathews S.A., Timms P. Improved prediction of bacterial transcription start sites // Bioinformatics. - 2006. - V. 22. - P. 142-148.

23. Knudsen S. Promoter2.0: for the recognition of PolII promoter sequences // Bioinformatics. - 1999. - V. 15. - P. 356-361

24. Reese M.G. Application of a time-delay neural network to promoter annotation in the Drosophila melanogaster genome // Comput Chem. - 2001. - V. 26. - P. 51-56.

25. Li Q.Z., Lin H. The recognition and prediction of sigma70 promoters in Escherichia coli K-12 // J Theor Biol. - 2006. V. 242. - P. 135-141.

26. Rangannan V., Bansal M. Relative stability of DNA as a generic criterion for promoter prediction: whole genome annotation of microbial genomes with varying nucleotide base composition // Mol Biosyst. - 2009. - V. 5. - P. 1758-1769.

27. Solovyev V., Salamov A. Automatic annotation of microbial genomes and metagenomic sequences // In: Metagenomics and its applications in agriculture, biomedicine and environmental studies. (Ed. R.W. Li), Nova Science Publishers. - 2011. - P. 6178. 
28. Song K. Recognition of prokaryotic promoters based on a novel variable-window Z-curve method // Nucleic Acids Res. 2012. - V. 40. - P. 963-971.

29. Hieno A., Naznin H.A., Hyakumachi M., Sakurai T., Tokizawa M. et al. ppdb: plant promoter database version 3.0 // Nucleic Acids Res. - 2014. - V. 42. - P. D1188-D1192.

30. Mitschke J., Vioque A., Haas F., Hess W.R., Muro-Pastor A.M. Dynamics of transcriptional start site selection during nitrogen stress-induced cell differentiation in Anabaena sp. PCC7120 // Proc Natl Acad Sci USA. - 2011. - V. 108. - P. 20130-20135.

31. Vijayan V., Jain I.H., O'Shea E.K. A high resolution map of a cyanobacterial transcriptome // Genome Biol. - 2011. - V. 12. P. R47.

32. Mitschke J., Georg J., Scholz I., Sharma C.M., Dienst D. et al. An experimentally anchored map of transcriptional start sites in the model cyanobacterium Synechocystis sp. PCC6803 // Proc Natl Acad Sci USA. - 2011. - V. 108. - P. 2124-2129.

33. Kilic S., White E.R., Sagitova D.M., Cornish J.P., Erill I. CollecTF: a database of experimentally validated transcription factorbinding sites in Bacteria // Nucleic Acids Res. - 2014. - V. 42. - P. D156-160.

34. Cardon L., Stormo G. Expectation maximization algorithm for identifying protein-binding sites with variable lengths from unaligned DNA fragments // J. Mol. Biol. - 1992. - V. 5. - P. 159-170.

35. Afifi A.A., Azen S.P. Statistical analysis: a computer oriented approach. Academic Press. - 2014. - 384 p.

36. Shahmuradov I.A., Umarov R.Kh., Solovyev V.V. TSSPlant: a new tool for prediction of plant Pol II promoters // Nucleic Acids Research. - 2017. - Published online 13 Jan 2017. doi: https://doi.org/10.1093/nar/gkw1353.

37. Shahmuradov I.A., Mohamad Razali R., Bougouffa S., Radovanovic A., Bajic V.B. bTSSfinder: a novel tool for the prediction of promoters in Cyanobacteria and Escherichia coli. Bioinformatics. - 2016. - Published online 30 Sep. doi: 10.1093/bioinformatics/btw629.

38. Reese M.G., Harris N.L., Eeckman F.H. Large Scale Sequencing Specific Neural Networks for Promoter and Splice Site Recognition. Biocomputing: Proceedings of the 1996 Pacific Symposium (edited by Lawrence Hunter and Terri E. Klein), World Scientific Publishing Co, Singapore, 1996, January 2-7.

39. Prestridge D.S. Predicting Pol II promoter sequences using transcription factor binding sites // J Mol Biol. - 1995. - V. 249. P. 923-932.

40. Abeel T., Saeys Y., Bonnet E., Rouze P., Van de Peer Y. Generic eukaryotic core promoter prediction using structural features of DNA // Genome Research. - 2008. - V. 18. - P. 310-323.

\section{SHAHMURADOV I.A.}

Institute of Molecular Biology and Biotechnologies, ANAS,

Azerbaijan, AZ1073, Baku, Matbuat Ave, 2a, e-mail: ilhambaku@gmail.com

\section{NOVEL TOOLS FOR THE PREDICTION OF PROMOTERS IN PLANTS AND BACTERIA}

Aim. The computational search for promoters remains an attractive problem in bioinformatics. Despite the attention it has received for many years, the problem has not been addressed satisfactorily. These studies were aimed to develop novel computer tools for prediction of promoters (transcription start sites, TSSs) in plants and bacteria. Results. Two novel tools for prediction of RNA polymerase II promoters in plants (TSSPlant) and bacteria (bTSSfinder) have been developed. TSSPlant achieves significantly higher accuracy compared to the next best promoter prediction program for both TATA and TATA-less promoters; it is available to download as a standalone program at http://www.cbrc.kaust.edu.sa/download/. bTSSfinder predicts promoters for five classes of $\sigma$ factors in Cyanobacteria $\left(\sigma^{\mathrm{A}}, \sigma^{\mathrm{C}}, \sigma^{\mathrm{H}}, \sigma^{\mathrm{G}}\right.$ and $\left.\sigma^{\mathrm{F}}\right)$ and for five classes of sigma factors in E. coli $\left(\sigma^{70}, \sigma^{38}, \sigma^{32}, \sigma^{28}\right.$ and $\left.\sigma^{24}\right)$. Comparing to currently available tools, bTSSfinder achieves highest accuracy. bTSSfinder is available standalone and online at http://www.cbrc.kaust.edu.sa/btssfinder. Conclusions. To date, TSSPlant and bTSSfinder are most accurate promoter predictors in plants and bacteria, respectively.

Keywords: transcription, RNA polymerase, promoter, TSS, promoter prediction. 